This is a postprint version of the following published document: L. Ruiz; R. J. Durán; I. de Miguel; P. S. Khodashenas; J.-J. Pedreno-Manresa; N. Merayo; J. C. Aguado; P. Pavon-Marino; S. Siddiqui; J. Mata; P. Fernández; R. M. Lorenzo; E. J. Abril. Genetic Algorithm for Effective Service Mapping in the Optical Backhaul of $5 G$ Networks. In: 2018 20th International Conference on Transparent Optical Networks (ICTON). DOI: https://doi.org/10.1109/ICTON.2018.8473766

(C) 2018 IEEE. Personal use of this material is permitted. Permission from IEEE must be obtained for all other uses, in any current or future media, including reprinting/republishing this material for advertising or promotional purposes, creating new collective works, for resale or redistribution to servers or lists, or reuse of any copyrighted component of this work in other works. 


\title{
Genetic Algorithm for Effective Service Mapping in the Optical Backhaul of 5G Networks
}

\author{
L. Ruiz ${ }^{1}$, R.J. Durán ${ }^{1}$, I. de Miguel ${ }^{1}$, P. S. Khodashenas ${ }^{2}$, J.-J. Pedreno-Manresa ${ }^{3}$, N. Merayo ${ }^{1}$, \\ J.C. Aguado $^{1}$, P. Pavon-Marino ${ }^{3}$, S. Siddiqui ${ }^{2}$, J. Mata ${ }^{1}$, P. Fernández ${ }^{1}$, R.M. Lorenzo ${ }^{1}$ and E.J. Abril ${ }^{1}$ \\ ${ }^{1}$ Optical Communications Group. Universidad de Valladolid. Paseo de Belén, 15, 47011, Valladolid, Spain \\ 2 i2CAT Foundation, C/ Gran Capita, 2, 08034, Barcelona, Spain \\ ${ }^{3}$ Universidad Politécnica de Cartagena, Cuartel de Antiguones, Plaza del Hospital 1, 30202 Cartagena, Spain \\ e-mail:lruiper@ribera.tel.uva.es,rduran@tel.uva.es
}

\begin{abstract}
A genetic algorithm is proposed to map virtual network functions in computing resources over $5 \mathrm{G}$ networks with an optical backhauling system. The algorithm outperforms other proposals in terms of blocking ratio and active CPU cores utilization.
\end{abstract}

Keywords: Network, network optimization, optical network.

\section{INTRODUCTION}

Future 5G technologies are expected to overcome the challenges of next generation communication systems, targeting to tackle the novel and manifold business requirements associated with distinct vertical industrial sectors. Extraordinarily high speeds and capacity, multi-tenancy, heterogeneous technologies convergence, on-demand service-oriented resource allocation, and coordinated automation, are only few examples of the complex demands $5 \mathrm{G}$ aims to undertake. The backhaul of those networks will be based on optical technologies due to their capacity and flexibility. Moreover, the shift from centralized cloud computing-based services towards data processing at the edge between the optical and radio segment is becoming one of the fundamental components envisaged to enable those future $5 \mathrm{G}$ technologies. Mobile edge computing (MEC) is focused on pushing processing to the optical network edge where all the actual interactions in the access networks take place and the critical low-latency processing occurs.

The landscape of future communication is reshaped and redefined significantly by the ongoing softwarization trends [1]. One of the main pillars of such revolution is the way that new network functions are introduced to the value chain. Traditionally, such process demands deployment of specialized devices with 'hard-wired' functionalities. It implies that any adaptation to the ever increasing and heterogeneous market requirements demands a huge investment to change/deploy hardware. Thanks to the advent of cloud computing, software defined networking (SDN) [2] and network function virtualization (NFV) [3], the idea of having general-purpose computing and storage assets at networks has been realized along with the softwarization of network functionalities, which enables the automation of network service provisioning and management.

Future internet will mainly use 5G in the access segment and a flexible optical technology for backhaul networks. Furthermore, the computing resources will not only be placed in central office $(\mathrm{CO})$ and data centers (i.e., following a cloud computing paradigm) but also in the optical network edges allowing the implementation of more distributed computing paradigms like MEC.

In this kind of networks, service deployment is the first step in the service lifecycle management. By definition, it consists of two main steps. The first one is service mapping, a logical process to decide where to deploy network service components (NS), i.e., virtual network functions (VNFs) over the MEC environment. This process will depend on parameters such as quality of service/experience $(\mathrm{QoS} / \mathrm{E})$ required by each service, resource availability, agreed service level agreement (SLA) with tenants, traffic patterns and predictions, energy efficiency, etc. Then, each service offered by the operator (e.g. VoIP, video, web services) will require a service chain (SC) composed of a set of VNFs to be traversed in a specific order. A service blocking occurs when not enough networking or computing resources to instantiate and connect all the VNFs that compose the $\mathrm{SC}$ are available. A wide range of solutions might be adopted to solve the service mapping problem, ranging from accurate Integer Linear Programming (ILP) formulations to heuristic algorithms. The second step is the deployment mechanism, which represents the actual interaction within virtual appliances such as NFV orchestrator (NFVO), virtual network function manager (VNFM), virtual infrastructure manager (VIM) to deploy VNFs.

There are several studies available in the literature focusing on VNF placement. In particular, Lin et al. modelled the resource allocation problem with a mixed-integer program and proposed a solution to incorporate limited physical resources into the NFV resource allocation [4]. Authors in [5] studied the resource allocation problem, considering the scalability issue, which might be caused by hosting multiple VNFs over the same hardware. Last but not least, in [6] authors studied the impact of latency requirements on the placement of VNFs constituting an added value service in a static scenario. ONOFRE project (Optical Network Convergence of the Future Internet) proposed in [7], [8] an algorithm for service chaining allocation in a real-life scenario, with a very 
high density of population and offered traffic expectations changing rapidly; with the aim of reducing service blocking ratio due to either unavailability of optical network capacity or computing resources.

In this paper, we also address the problem of service mapping in a 5G network with different macro-cells and micro-cells considering the optical network and the computing resources in the optical network edge nodes (to provide MEC) and in CO. We propose a genetic algorithm that looks for (i) available hardware resources to instantiate the VNFs that compose the SC associated to the demanded service, and (ii) available bandwidth in the optical network connecting the cells expected to host the VNFs. In contrast with those previous studies that only minimize the service blocking ratio, the proposed algorithm minimizes this parameter but also reduces the CPU usage (and therefore, the energy consumption and operating expenses -OPEX-).

\section{A GENETIC ALGORITHM TO SOLVE THE SERVICE MAPPING PROBLEM}

Genetic algorithms are a family of computational models that encode the solution to a problem in a chromosomelike data structure (also called individual) and apply recombination operations so that they preserve critical information [9]. In our proposal, genetic algorithm for service mapping (GASM), each potential solution to the VNF allocation problem is characterized by a chromosome composed by a number of genes. Each gene represents the number of instances of a given VNF hosted in a location, be it a CO or MEC in the optical network edge nodes. When chromosomes are translated into a solution, the algorithm creates the number of VNF instances in each location that the genes indicate. After that, user demands are sorted by the priority defined by the operator (e.g., first serve VoIP demands, then video and finally web demands). Then, these demands are served by assigning available VNFs to them. The VNFs that must be assigned are given by the corresponding SC associated to each service. A VNF is available if it processes less operations than its maximum number of concurrent operations per instance. The algorithm looks for VNFs at the local cell first, and only searches for VNFs in the CO when no available VNF are present at the local cell, like in [7], [8]. Once the algorithm finds an available VNF of the SC in the CO, it will not look for VNFs at MEC nodes again. The service is blocked if the algorithm is unable to set up the SC either due to lack of VNF resources or bandwidth availability in the optical network.

Two fitness parameters are computed for each potential solution of the genetic algorithm (i.e., for each individual or chromosome): the service blocking ratio and the percentage of required active CPU cores (as a way to reduce the energy consumption). In order to improve the performance, two ad-hoc individuals are created in the first generation of the genetic algorithm together with other randomly generated ones. The first ad-hoc individual is created so that its solution is equivalent to following the policy used in [7], [8]. We call this policy "MEC First" since it first tries to instantiate or use VNFs at the radio node. Only when the IT resources are insufficient or the existing VNFs are not reusable, does the algorithm look for resources at the CO. The second ad-hoc individual is called "CO First", because the chromosome provides a solution equivalent to trying to instantiate/use VNFs at the $\mathrm{CO}$ first, and then at the local radio cell. We consider all radio cells to be MEC nodes.

GASM employs classic genetic operators (i.e., crossover and mutation) to create new populations. When a new individual is created using these operators, GASM checks it is a valid one considering the instantiated VNFs and the available computing resources (CPU, RAM and HDD) in the nodes and CO. An individual is not valid if the algorithm cannot instantiate al the VNFs indicated by the genes. When this occurs, GASM discards individual and creates a new one. GASM generates new individuals by crossover/mutation until completing the desired population size. The fitness parameters of the individuals of the new population (service blocking ratio and percentage of active CPU cores) are computed as previously mentioned. Then, the individuals with lowest service blocking ratio are selected to survive in the next generation. If needed, ties are broken by selecting the individual with less active CPU cores. The algorithm repeats the process for as many generations as defined by the user.

\section{SIMULATION STUDY AND RESULTS}

A simulation study has been conducted in $\mathrm{OMNeT}++(\mathrm{C}++$ based discrete event simulator $)$ in order to evaluate the performance of GASM. We have selected a star access network topology populated with two types of nodes: 10 macro-cells and 10 micro-cells. Both types of cells are connected to a CO via $10 \mathrm{Gbps}$ optical links. The nodes are equipped with the computational resources shown in Table 1 like in $[7,8]$. We assume that all the nodes are NFV-enabled. Each service has an associated SC and bandwidth requirements that can be found in Table 2. Table 3 shows the hardware requirements and the maximum number of concurrent operations associated to an instance of each VNF.

We define the input parameter $\bar{u}$ as the average number of users per macro-cell. At the beginning of each simulation, the number of users that are connected to each macro-cell is randomly chosen using a uniform distribution between $[0,2 \bar{u}]$ (i.e., different number in each macro-cell). The average number of users connected to each micro-cell is considered to be the $10 \%$ of that of macro-cells, i.e., the number of users in each micro-cell is generated randomly using a uniform distribution between $[0,2 \bar{u} / 10]$. Each user can request a service, either VoIP, Video or Web Service, with a probability of $30 \%, 20 \%$ or $50 \%$ respectively [8]. We repeated the simulation 100 times with different traffic demands. All figures are plotted with $95 \%$ confidence intervals. 
Table 1. Hardware capabilities of the different nodes

\begin{tabular}{|l|l|}
\hline Location & Computational resources \\
\hline Central Office & 100 CPU cores, 480 GB RAM and 27 TB HDD \\
\hline Macro-cell & 16 CPU cores, 64 GB RAM and 10 TB HDD \\
\hline Micro-cell & 8 CPU cores, 32 GB RAM and 7 TB HDD \\
\hline
\end{tabular}

Table 2. Requirements of the deployed service chains

\begin{tabular}{|l|l|r|}
\hline Service & Chained VNF** & Bandwidth \\
\hline VoIP & NAT-FW-TM-FW-NAT & $64 \mathrm{kbps}$ \\
\hline Video & NAT-FW-TM-VOC-IDPS & $4 \mathrm{Mbps}$ \\
\hline Web Services & NAT-FW-TM-WOC-IDPS & $100 \mathrm{kbps}$ \\
\hline
\end{tabular}

*NAT:Network Address Translator, FW: Firewall, TM: Traffic Monitor, WOC: WAN Optimization Controller, VOC: Video Optimization Controller, IDPS: Intrusion Detection Prevention System [5]-[8].

Table 3. Hardware requirements associated to the VNFs [5]-[8]

\begin{tabular}{|c|l|c|}
\hline Service & \multicolumn{1}{|c|}{ HW requirements. } & \# concurrent operations \\
\hline NAT & CPU: 1 core, RAM: 1 GB, HDD: 2 GB & 3000 \\
\hline FW & CPU: 2 cores, RAM: 3 GB, HDD: 5GB & 2500 \\
\hline TM & CPU: 1 core, RAM: 3 GB, HDD: 2 GB & 2500 \\
\hline VOC & CPU: 2 cores, HDD: 20GB, RAM: 2GB & 1000 \\
\hline WOC & CPU: 1 core, HDD: 10GB, RAM: 2GB & 1500 \\
\hline IDPS & CPU: 2 cores, HDD: 10GB, RAM: 2GB & 2500 \\
\hline
\end{tabular}

Figure 1 shows the service blocking ratio with GASM and the other two methods depending on the average number of users per macro-cell. Figure 2 shows the corresponding values of percentage of active CPU cores. These figures show that GASM achieves the same service blocking ratio as the "MEC First" policy [7], [8] (Figure 1), but reducing the number of active CPU cores (and thus energy consumption) when compared to this policy (a reduction ranging from 45 to almost 10 percentage points, depending on the number of users, as shown in Figure 2) for up to 4,500 average users. When compared to the "CO First" policy, the latter requires the same or less number of active CPU cores than GASM, but GASM significantly improves the results in terms of blocking ratio. Hence, GASM maps the services in the network computing resources achieving a very good trade-off in terms of blocking ratio and number of active CPU cores, improving the results previously published in [7], [8].

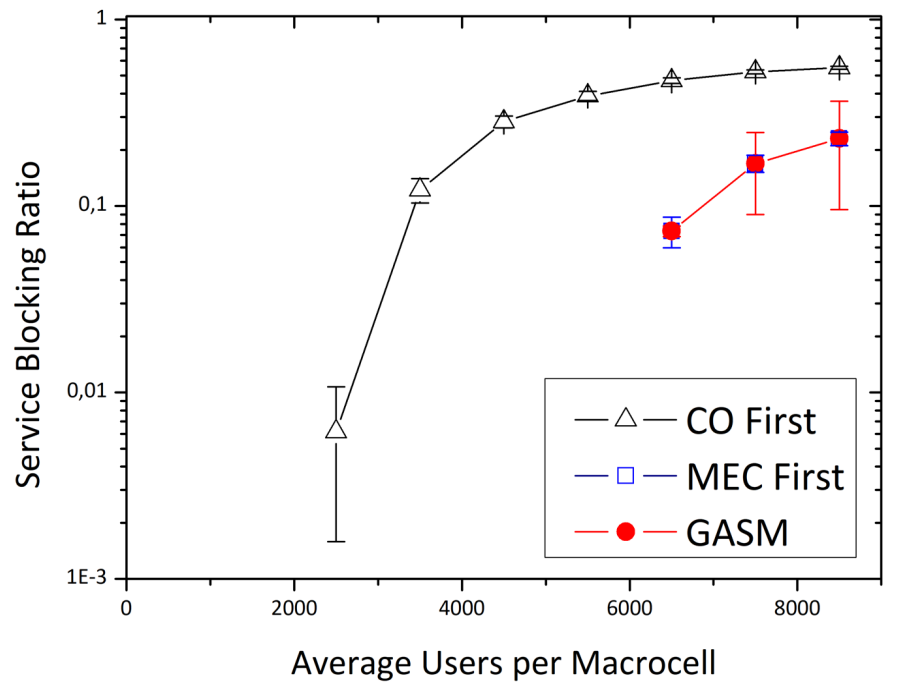

Figure 1. Service blocking ratio. 


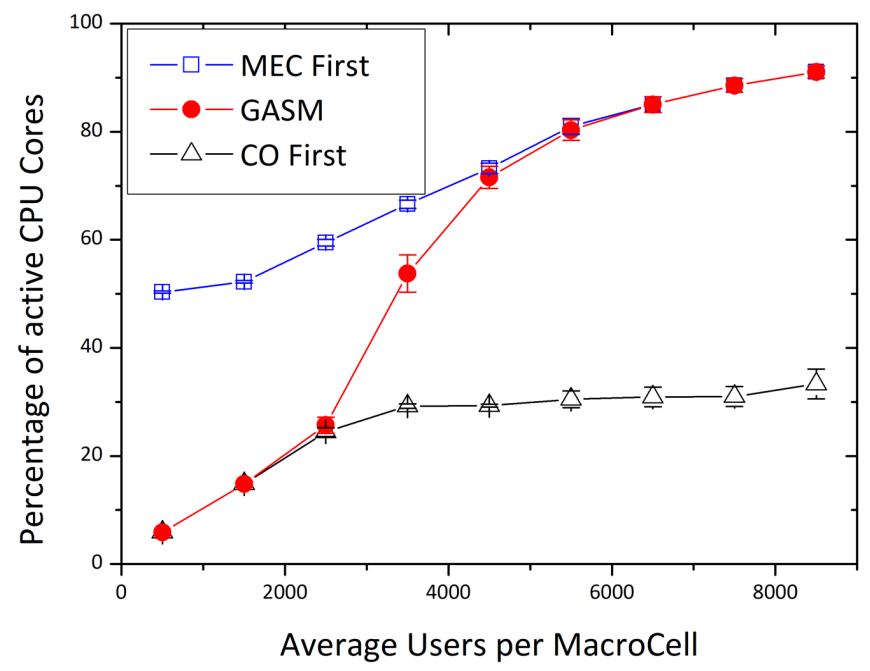

Figure 2. Percentage of active CPU cores.

\section{CONCLUSIONS}

In this paper, we presented GASM, a genetic algorithm to solve the problem of service mapping in an NVF-enabled 5G access optical network. We compare our proposal with two service mapping policies, "MEC First" and "CO First". The results showed that GASM provides an excellent trade-off between the reduction of blocking ratio and the reduction of active CPU cores in the network (and, therefore, in energy consumption and OPEX).

\section{ACKNOWLEDGEMENTS}

This work has been supported by Spanish Ministry of Economy and Competitiveness (TEC2014-53071-C3-2-P, TEC2017-84423-C3-1-P, TEC2015-71932-REDT), the fellowship program of the Spanish Ministry of Education, Culture and Sports (BES-2015-074514 and FPU14/04227) and European H2020-ICT-2014-2 SESAME (no. 671596) and H2020-ICT-2016-2 METRO-HAUL (no. 761727).

\section{REFERENCES}

[1] European Commission, The 5G Infrastructure Public Private Partnership: the next generation of communication networks and services, in 5G Vision, (Retrieved: June 23, 2016).

[2] ETSI NFV white paper, Network Function Virtualisation: An Introduction, Benefits, Enablers, Challenges and Call for Action, 2012.

[3] R. Jain and S. Paul, Network Virtualization and Software Defined Networking for Cloud Computing - A Survey, IEEE Communications Magazine, vol. 51, no. 11, pp. 24-31, Nov. 2013.

[4] T. Lin et al.: Optimal Network Function Virtualization Realizing End-to-End Requests, in Proc. IEEE GLOBECOM, San Diego, U.S.A., Dec. 2015.

[5] M. Savi et al.: Impact of Processing Costs on Service Chain Placement in Network Functions Virtualization, in Proc. IEEE NFV-SDN, San Francisco, U.S.A., Nov. 2016.

[6] M. Savi et al:: To distribute or not to distribute? Impact of latency on Virtual Network Function Distribution at the edge of FMC Networks, in Proc. ICTON 2016, Trento, Italy, Jul. 2016.

[7] J.-J. Pedreno-Manresa et al.: Dynamic QoS/QoE assurance in realistic NFV-enabled 5G Access Networks, in Proc. ICTON 2017, Girona, Spain, Jul. 2017.

[8] J.-J. Pedreno-Manresa et al.: On the Need of Joint Bandwidth and NFV Resource Orchestration: a Realistic 5G Access Network Use Case, IEEE Communications Letters, 2018, vol. 22, no 1, pp. 145-148, Jan. 2018.

[9] D. Whitley, A genetic algorithm tutorial, Statistics and computing, vol. 4, no 2, pp. 65-85, Jun. 1994. 